\title{
MiR-744 mediates the oxaliplatin chemoresistance in colorectal cancer through inhibiting BIN1
}

\author{
Y. ZHOU, A. HE, L. ZHANG, G. YI \\ Department of General Surgery, Xian No.1 Hospital, Northwest University, Shaanxi, China \\ ${ }^{*}$ Correspondence: yigangfeng@163.com
}

Received May 8, 2019 / Accepted July 11, 2019

\begin{abstract}
Colorectal cancer (CRC) is one of the most common malignant cancers worldwide. However, lacking of novel and sensitive chemotherapy revealed the major challenge to improve the survival of CRC patients. The aim of this study was to explore the effect and mechanism of miR-744 on the oxaliplatin chemoresistance in CRC. Firstly, the levels of miR-744 were elevated significantly in CRC tissues from patients with oxaliplatin administration before surgery and in oxaliplatin-resistant HCT116 cells. Then, the oxaliplatin chemoresistance was enhanced by miR-744 overexpression, while was attenuated by miR-744 inhibition in HCT116 and T84 cells. Additionally, the level of BIN1 protein was found to be regulated negatively by miR-744, and BIN1 overexpression blocked the oxaliplatin chemoresistance induced by miR-744. Furthermore, BIN1 was proved to be a direct target of miR-744 by luciferase reporter assay. Taken together, these findings demonstrated that miR-744 might positively mediate the oxaliplatin chemoresistance through suppressing BIN1 expression in CRC cells, thus suggested a rationale target for the developing more effective strategies to reverse oxaliplatin resistance in CRC treatment.
\end{abstract}

Key words: miR-744, oxaliplatin, colorectal cancer, BIN1

Colorectal cancer (CRC) is one of the most malignant cancer types around the world due to its high morbidity and mortality $[1,2]$. The patients' poor prognosis associated with CRC are partially due to resistance to existing therapies; despite the in-depth studies of the molecular mechanisms underlying colon cancer and improvements in the multidisciplinary and comprehensive treatment [3], our understanding of the mechanisms underlying therapy resistance, as well as the number of available therapeutic options, remains limited. Recently, it has been reported that the aberrant miRNAs expression has contributed to the chemoresistance of cancer.

RNA molecules which do not encode proteins are called non-coding RNAs (ncRNAs), and people have witnessed a growing understanding about the various function of microRNAs, a subgroup of small ncRNAs, in recent decades. MicroRNAs act as transcriptional repressors by interacting with the 3'UTR regions of their downstream target genes. MicroRNAs are known to be involved in many cellular processes, and their dysregulation is found in various human malignancies [4]. MicroRNAs play critical roles in tumorigenesis, cell proliferation and metabolism and programmed cell death $[5,6]$. As these biological processes are pertinent closely to chemosensitivity and chemoresistance, it is hypothesized that microRNAs could play an important role in modulating response toward anticancer drugs. MiR-744 is reported to be dysregulated in many carcinoma tissues. MiR-744 was upregulated in malignant head and neck cancer lesions compared to normal tissues [7]. High level of plasma miR-744 contributed to chemoresistance and poor prognosis of pancreatic cancer patients who underwent gemcitabinebased chemotherapy [8,9]. Besides, the level of serum miR-744 was upregulated in gastric cancer patients and showed an increasing trend during GC development [10]. These findings suggest that miR-744 plays a possible role in chemoresistance of CRC.

In the current study, we revealed that oxaliplatin, a basic drug of CRC adjuvant chemotherapy, enhanced the expression of miR-744 in human CRC cell lines. Besides, overexpression of miR-744 reduced the cell death when exposed to oxaliplatin. Furthermore, we identified that Bridging integrator 1 (BIN1) was a direct target of miR-744. BIN1 is a widely investigated tumor suppressor reported to inhibit c-MYC-mediated transactivation and transformation [11, 12] and to be involved in the response to DNA damage of cancer cells [13]. BIN1 is a predicted target of miR-744 in the Targetscan software. Hence, we propose that miR-744 inhibits the expression of BIN1, by which exerts the chemoresistance effect on colorectal cancer cells. 


\section{Materials and methods}

Cell culture. Human CRC cell lines HCT116 and T84 cells were purchased from the Shanghai Institute of Biochemistry and Cell Biology, Chinese Academy of Sciences (Shanghai, China). Cell lines were cultivated in complete Dulbecco's modified Eagle's medium/F12 (DMEM/F12, Invitrogen Argentina SA) supplemented with $10 \%$ ( $\mathrm{vol} / \mathrm{vol}$ ) fetal bovine serum (FBS, NatoCor, Argentina), $50 \mathrm{U} / \mathrm{ml}$ of penicillin G and $50 \mathrm{mg} / \mathrm{ml}$ of streptomycin sulfate in a humidified atmosphere with $5 \% \mathrm{CO}_{2}$ at $37^{\circ} \mathrm{C}$.

To establish oxaliplatin resistant HCT116 cell line, $5 \mathrm{uM}$ oxaliplatin was used to treat HCT116 cells which caused $50 \%$ cell death. HCT116 cells were exposed to $5 \mu \mathrm{M}$ oxaliplatin for $48 \mathrm{~h}$ to reach $80 \%$ confluence and passaged twice in this concentration of oxaliplatin. Then, the concentration of oxaliplatin was gradually increased by $1 \mu \mathrm{M}$ with each subculture of HCT116 cells. After subculture and incubation with oxaliplatin solution was repeated about 35 times, the HCT116 cells resistant to $40 \mu \mathrm{M}$ oxaliplatin were obtained. Meanwhile, the oxaliplatin resistant HCT116 cell line was cultured continuously in complete culture medium containing $5 \mu \mathrm{M}$ oxaliplatin.

Tissue samples. CRC tissues were collected from patients who underwent surgical resection at Xian No. 1 Hospital from May 2016 to May 2017. All patients signed consent letters and all manipulation of the tissues was approved by the Ethics Committee of Xian Jiaotong University. After surgery, the tissue samples were immediately frozen in liquid nitrogen and stored at $-80^{\circ} \mathrm{C}$. All experiments were performed in accordance with The Code of Ethics of the World Medical Association (Declaration of Helsinki) and the guidelines of the Xi'an Jiaotong University.

Vector constructs and cell transfection. Hsa-miR-744 mimic, inhibitor and negative control were purchased from Ribo Biotech (Guangzhou, China). The sequence of miR-744 mimic was 5'-UGCGGGGCUAGGGCUAACAGCA-3'. Human BIN1 gene was inserted into pcDNA3 vectors (Invitrogen, Carlsbad, CA) to construct BIN1 plasmid. For overexpression of miR-744, the miR-744 mimic or control mimic was used to transfect HCT116 and T84 cells with a final concentration of $10 \mathrm{mM}$, using Lipofectamine LTX (Invitrogen) according to the manufacturer's instructions. After $24 \mathrm{~h}$, the overexpression of miR-744 was confirmed by qRT-PCR using the human TaqMan MicroRNA Assay Kit (Applied Biosystems). To inhibit miR-744 or overexpress BIN1, the same method was conducted.

Real-time PCR. Real-time PCR was performed to detect the expression level of miR-744 in CRC cancer tissue and cell. Briefly, TRIzol (Invitrogen; Thermo Fisher Scientific, Inc.) was used to isolate total RNA from tissues and cells. Complementary DNA (cDNA) was synthesized from $100 \mathrm{ng}$ of total RNA using a TaqMan MicroRNA Reverse Transcription Kit (Applied Biosystems, Foster City, CA, USA) and it served as the template for amplification of PCR using a TaqMan MicroRNA
Assay kit (both from Applied Biosystems) on the ABI 7900 Fast System. The PCR conditions were $95^{\circ} \mathrm{C}$ for $5 \mathrm{~min}$, followed by 40 cycles at $95^{\circ} \mathrm{C}$ for $15 \mathrm{~s}, 54^{\circ} \mathrm{C}$ for $30 \mathrm{~s}$ and $72^{\circ} \mathrm{C}$ for $34 \mathrm{~s}$. Each sample was examined in triplicate and RNU6B was used as internal control for normalization. Real-time PCR primers: miR-744: F 5'-AATGCGGGGCTAGGGCTA-3'; R: 5'-GTGCAGGGTCCGAGGT-3'; U6: F: 5'-GCGCGTCGTGAAGCGTTC-3'; R: 5'-GTGCA-GGGTCCGAGGT-3'

Luciferase reporter system. The luciferase reporter assay was used to examine whether the 3'-UTR of BIN1 is a target of miR-744. The wild-type (wt) and mutated (mut) miR-744 putative targets on BIN1 3'UTR were cloned into pGL-3 luciferase reporter vector. Transfection was carried out using Lipofectamine LTX and the Plus reagent (Invitrogen) as recommended. Cells were seeded in the absence of serum for $2 \mathrm{~h}$ prior to transfection with the luciferase reporter constructs together with miR-744 plasmid. The firefly and renilla luciferase activity were determined by the dual luciferase reporter assay kit (Promega) and luciferase was measured on a Fluostar Optima luminometer.

Western blot. Proteins were separated on a $12 \%$ SDS-PAGE gel and transferred onto a poly-vinylidene fluoride (PVDF) membranes (Millipore, Billerica, MA). The membrane was blocked with $5 \%$ non-fat milk and incubated with anti-BIN1 antibody or anti-beta-actin antibody (Sigma, CA, USA). After being washed three times in TBST for $5 \mathrm{~min}$ each, membranes were incubated with the HRP-conjugated secondary antibodies (1:5000) subsequently and followed by three times washing. The proteins were detected using enhanced chemiluminescence reagents by X-ray films.

TUNEL assay. The terminal deoxynucleotidyl transferase dUTP nick end labeling (TUNEL) assay allows quick and easy demonstration of cell apoptosis. The Click-iT Plus TUNEL Assay for In Situ Apoptosis Detection Kit (Roche, Shanghai, China) was utilized to stain nuclei of apoptotic cells. The experiment was performed according to the protocol provided by the manufacturer. The ratio of TUNEL positive cells was recorded.

CCK-8 assay. Chemosensitivity was measured using Cell Counting Kit-8 (CCK-8, Beyotime, Shanghai, China). The cells were seeded in a 96-well plate and grown in DMEM/ F12 for $24 \mathrm{~h}$. Then, cells were treated with $15 \mu \mathrm{M}$ oxaliplatin for $24 \mathrm{~h}$. Ten $\mu \mathrm{l}$ per well of CCK8 reagent was added and the plate was incubated at $37^{\circ} \mathrm{C}$ for $3 \mathrm{~h}$. The optical density (OD) was measured at an absorbance of $450 \mathrm{~nm}$. All assays were performed in triplicate.

Statistics analysis. Results represent mean \pm standard deviation of three independent experiments. Two-sided $\mathrm{t}$-test was applied to evaluate differences between two groups and one-way ANOVA with Tukey's Multiple Comparisons adjustments for multiple comparisons. The replicate samples were analyzed a minimum of three times. All calculations were performed by GraphPad Prism 6 software (GraphPad Software, Inc., CA, USA). A p-value $<0.05$ was considered statistically significant. 


\section{Results}

Oxaliplatin induced elevated level of miR-744 in colorectal cancer tissues and cell line. We collected 24 colorectal cancer tissue samples from patients who had received oxaliplatin administration before surgery as well as 32 samples from the ones without chemotherapy. Real-time quantitative PCR (qRT-PCR) was used to detect the expression of miR-744. The level of miR-744 was much higher in tissues treated oxaliplatin chemotherapy than the un-treated ones (Figure 1A). Then, we established the oxaliplatin resistant HCT116 cell line (HCT116R cells) by the incremental concentration of oxaliplatin exposure for 3 months. The drug resistant effect of the HCT116R cells was assessed by CCK- 8 assay. HCT116R had a higher cell viability than the normal HCT116 cells after $15 \mu \mathrm{M}$ oxaliplatin treatment for 24 hours (Figure 1B). Moreover, the miR-744 level was higher in HCT116R cells after oxaliplatin treatment for 3 months compared with the normal HCT116 cells (Figure 1C). Meanwhile, the expression of miR-744 was increased until 75 days during 3 months oxaliplatin exposure (Figure 1D). This result suggests that the long term oxaliplatin administration induces chemoresistance of CRC cells and upregulates the expression of miR-744.

A

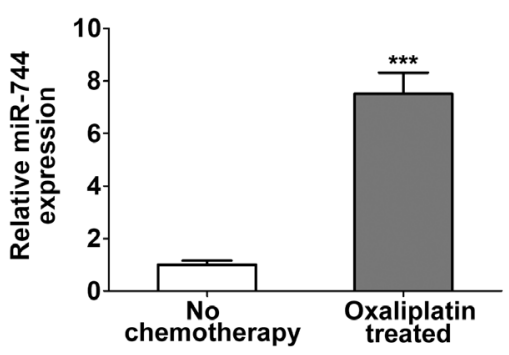

C

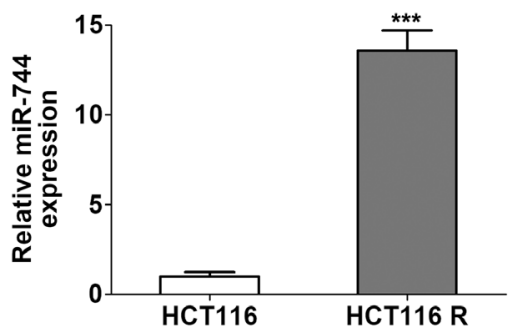

CRC cells transfected with miR-744 mimic showed a drug resistant character against oxaliplatin-induced cell death. MiR-744 mimic and inhibitor were transfected into HCT116 cells, T84 cells and HCT116R cells. qRT-PCR assay showed that miR-744 level was obviously increased in the mimic group and decreased in the inhibitor group in HCT116 and T84 cell lines (Figures 2A-C). Then, cells were treated with oxaliplatin for 12 hours and CCK- 8 assay was applied to detect the cell death. The results revealed that oxaliplatin induced less cell death in HCT116 cells transfected with miR-744 mimic than in cells transfected with control vectors (Figure 2D). The same result was found in T84 cells (Figure 2E). However, the cell viability in HCT116 cells transfected with miR-744 mimic didn't change significantly compared to the cells transfected with control vectors (Figure 2F). Conversely, oxaliplatin induced much more cell death when miR-744 inhibitor plasmid was transfected into HCT116, HCT116R and T84 cells (Figures 2D-F). These findings indicate that miR-744 mediates oxaliplatin-induced chemoresistance in CRC cells and miR-744 inhibition sensitizes CRC cells to oxaliplatin.

BIN1 was downregulated in oxaliplatin-resistant HCT116 cells and was a direct target of miR-744. BIN1 is a predicted target of miR-744 in the Targetscan software, which

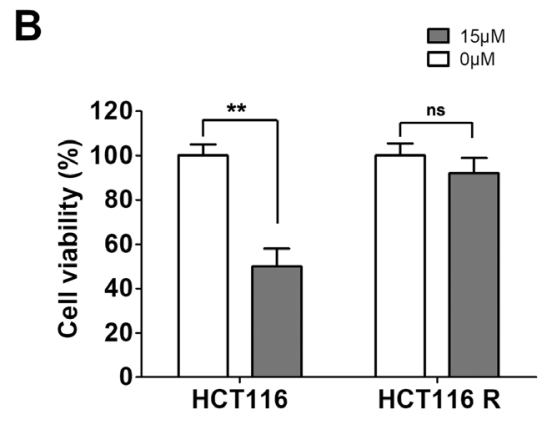

D

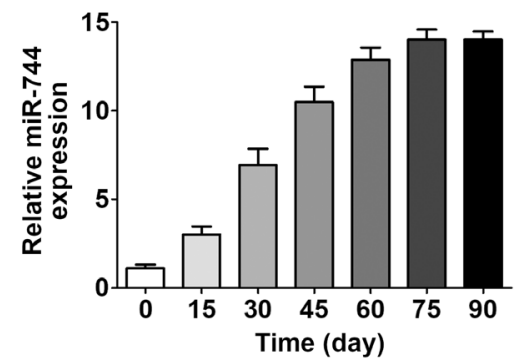

Figure 1. MiR-744 was increased in oxaliplatin resistant CRC tissue and cell. A) The level of miR-744 in colorectal cancer tissue samples from oxaliplatin treated patients and no chemotherapy treated patients. B) The CCK-8 assay was applied to detect the cell viability of normal HCT116 cells and the established oxaliplatin resistant HCT116 cells after treated with $15 \mu \mathrm{M}$ oxaliplatin. C) The level of miR-744 in normal HCT116 cells and the established oxaliplatin resistant HCT116 cells. D) The level of miR-744 in HCT116 cells over three months for oxaliplatin exposure. ${ }^{\star *}$ p $<0.01{ }^{\star * *}$ p $<0.001$; ns, no significance) 
is reported to be a tumor suppressor by inhibiting c-MYCmediated transactivation and transformation in cancer cells. Here, the expression of BIN1 was detected by western blot in HCT116 cells. The result showed that BIN1 was decreased in HCT116R cells than the normal HCT116 cells (Figure 3A). To further investigate the relation between miR-744 and BIN1, HCT116 cells were transfected with miR-744 mimic or inhibitor plasmid and then BIN1 was detected. It revealed that BIN1 was downregulated by miR-744 mimic and the expression of BIN1 increased when miR-744 was inhibited (Figure 3B). Furthermore, we performed the luciferase reporter assay by constructing luciferase reporter vector containing the 3'-UTR sequences of BIN1 with wildtype (wt) or mutant (mut) miR-744 binding sites. When we co-transfected HCT116 cells or T84 cells with miR-744 mimic and wildtype 3'-UTR sequences of BIN1, the luciferase activity was significantly suppressed (Figure 3C). Meanwhile, the luciferase activity of the mutant 3'-UTR of BIN1 was not affected. To further verify the relationship between BIN1 and miR-744, we co-transfected HCT116 or T84 cells with BIN1, wildtype (wt) and mutant (mut) miR-744. The luciferase reporter assay showed that the luciferase activity was significantly suppressed and the mutant miR-744 was not affected (Figure 3D). Hence, we revealed that BIN1 was a direct target of miR-744.

BIN1 blocked miR-744-induced oxaliplatin resistance in HCT116 cells. Although we proved that BIN1 was a direct target of miR-744, whether it mediated the miR-744-induced oxaliplatin resistance of HCT116 cells was still unknown. So HCT116 cells were treated with oxaliplatin and then
A

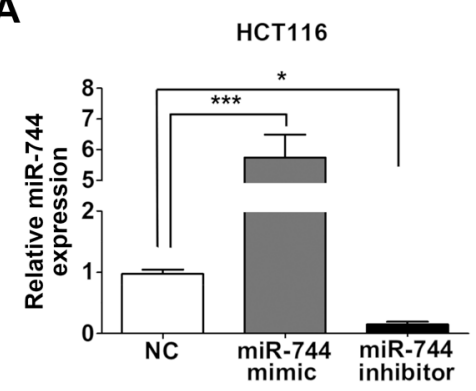

C

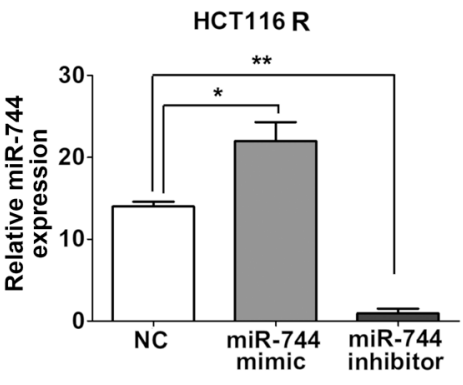

E

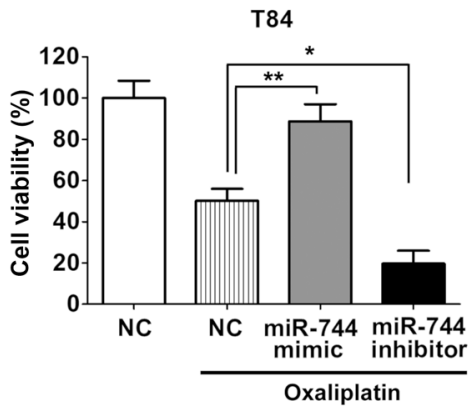

B

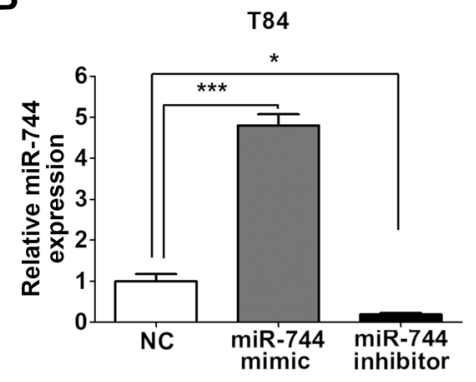

$\mathbf{D}$

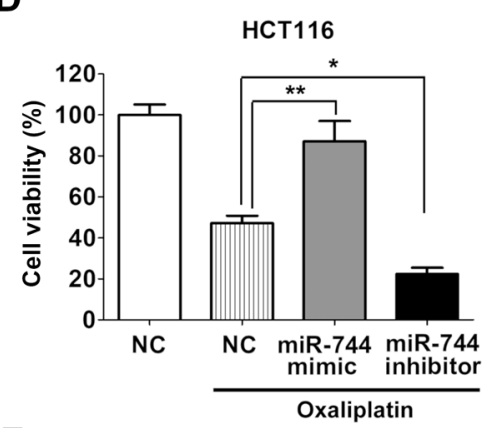

$\mathbf{F}$

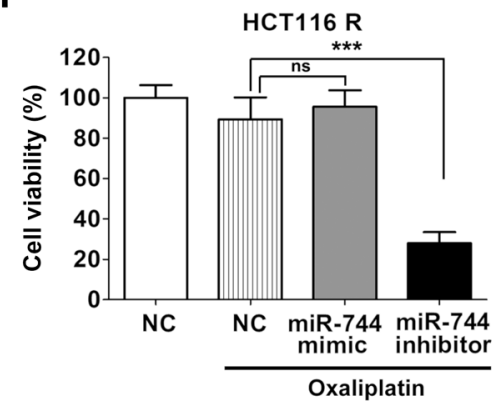

Figure 2. MiR-744 mediated the oxaliplatin resistance of CRC cell. A) The level of miR-744 in HCT116 cells transfected with NC, miR-744 mimic or miR-744 plasmid. B) The level of miR-744 in T84 cells transfected with NC, miR-744 mimic or miR-744 inhibitor plasmid. C) The level of miR-744 in HCT116R cells transfected with NC, miR-744 mimic or miR-744 inhibitor plasmid. D) Cell viability was detected in HCT116 cells transfected with NC, miR-744 mimic or miR-744 plasmid after treated with $15 \mu \mathrm{M}$ oxaliplatin. E) Cell viability was detected in T84 cells transfected with NC, miR-744 mimic or miR-744 plasmid after treated with $15 \mu \mathrm{M}$ oxaliplatin. F) Cell viability was detected in HCT116R cells transfected with NC, miR-744 mimic or miR-744 plasmid after treated with $15 \mu \mathrm{M}$ oxaliplatin. $\left({ }^{*} \mathrm{p}<0.05 ;{ }^{* *} \mathrm{p}<0.01 ;{ }^{* * *} \mathrm{p}<0.001\right.$; ns, no significance) 
A
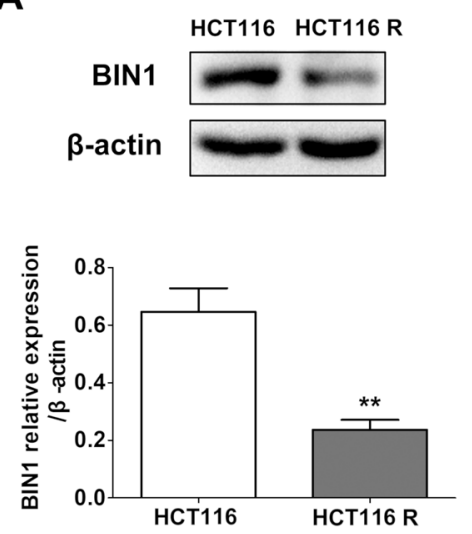

C
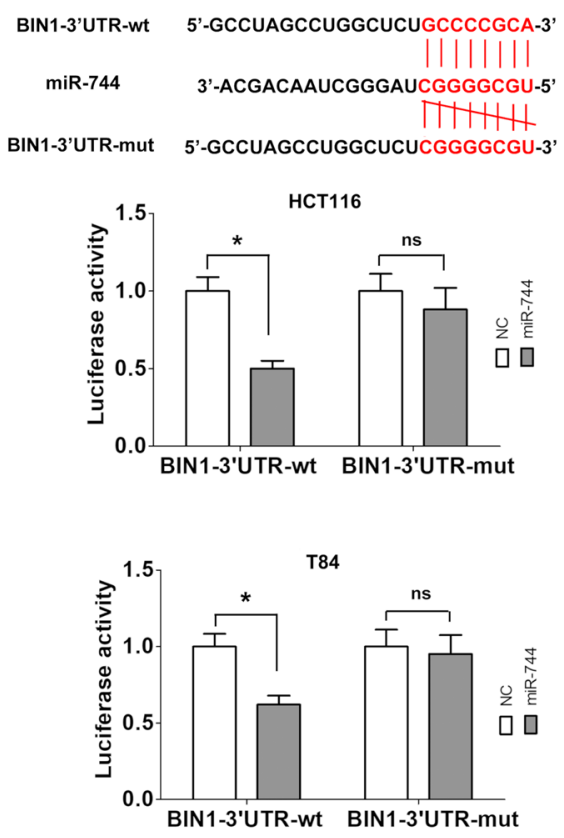
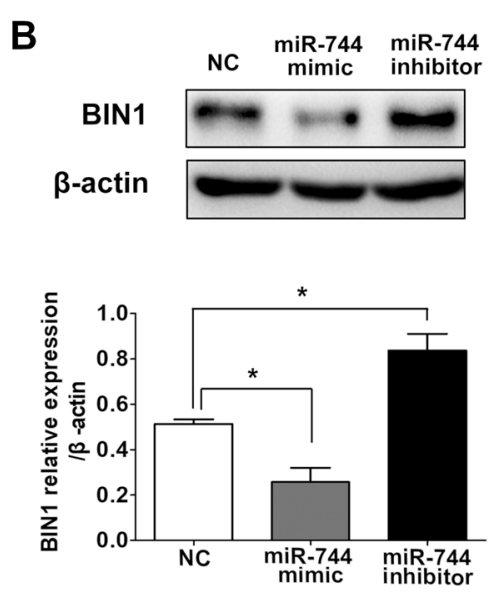

D
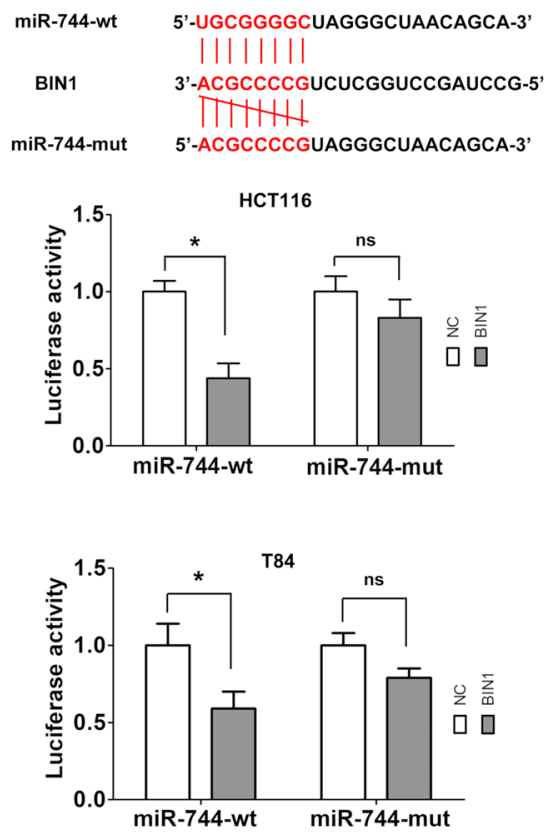

Figure 3. BIN1 could be regulated by miR-744 and was a direct target of miR-744. A) BIN1 was downregulated in the oxaliplatin resistant HCT116 cells. B) The protein level of BIN1 was downregulated by miR-744 mimic and upregulated by miR-744 inhibitor. C) The luciferase activity was suppressed in miR-744 mimic and wildtype 3'-UTR-BIN1 co-transfected HCT116 cells or T84 cells. D) MiR-744 did not affect the luciferase activity of mutant 3'-UTR-BIN1 transfected HCT116 cells or T84 cells. $\left({ }^{\star} \mathrm{p}<0.05 ;{ }^{* *} \mathrm{p}<0.01\right.$; ns, no significance)

co-transfected with miR-744 mimic or miR-744 inhibitor and BIN1 or siBIN1, mutant BIN1 plasmid. The oxaliplatinresistant effect mediated by miR-744 was weakened by BIN1 overexpression, which suggested that BIN1 was essential for oxaliplatin to induce the death of CRC cells (Figure 4A). Then, the TUNEL staining assay was applied to detect the cell death after oxaliplatin treatment. The result showed the cell viability increased significantly in the miR-744 group compared with the control group. However, overexpression of BIN1 can reverse such effect (Figure 4B). These results indicated that miR-744 mediated oxaliplatin resistance via downregulating BIN1.

\section{Discussion}

Therapeutic resistance is a major challenge in effective cancer treatment. Oxaliplatin resistance is currently one of the major obstacles in CRC chemotherapy. Although great progress has been made in cancer therapy, majority of clinically effective chemotherapy inducers are ineffective due to the complexity of the mechanisms underlying chemotherapy resistance. Development of chemotherapy sensitive or resistant biomarker assays for the prognosis estimation and designing more effective therapies of CRC patients is urgently required to reduce the high morbidity and 
A

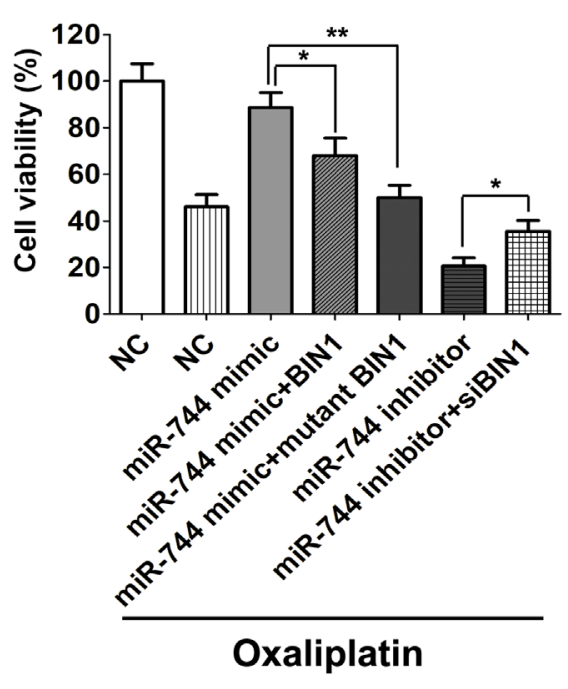

B

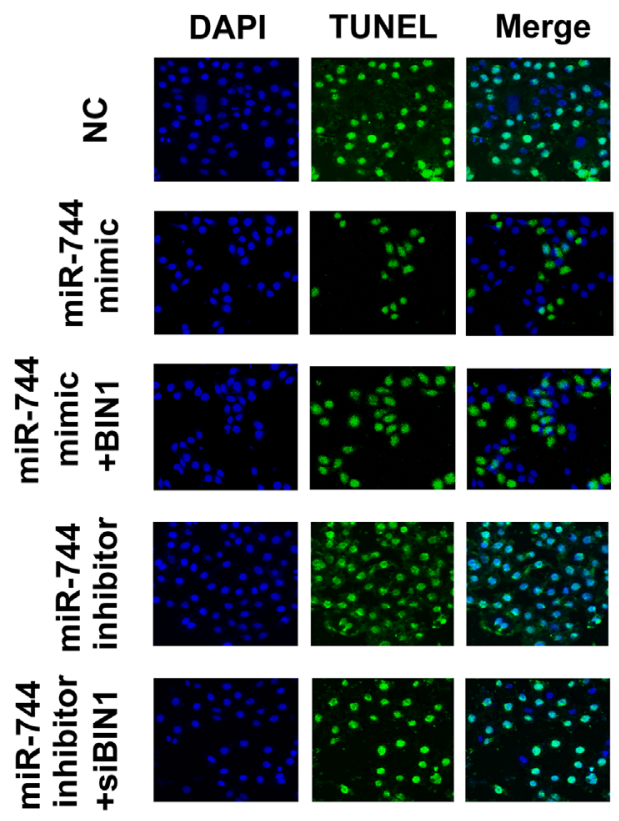

Figure 4. BIN1 mediated the miR-744 induced oxaliplatin resistance of CRC cell. A) CCK-8 assay revealed that BIN1 inhibited the oxaliplatin resistance effect of HCT116 cells induced by miR-744. B) Cell death of the transfected HCT116 cells after oxaliplatin treatment was detected by the TUNEL staining assay. $\left({ }^{*} \mathrm{p}<0.05 ;{ }^{* *} \mathrm{p}<0.01\right)$

mortality associated with this lethal disease. In recent years, many miRNAs have been identified as potential drug resistant biomarker of various cancers [14-16]. By monitoring the level of these miRNAs, we can evaluate the therapeutic effect of certain drugs on the tumor. Previous studies have identified several miRNAs contributed to the chemoresistance of colorectal cancer. For instance, miR-503-5p confers drug resistance by targeting p53 upregulated modulator of apoptosis (PUMA) in colorectal carcinoma [17]. MiR-506 enhances the sensitivity of human colorectal cancer cells to oxaliplatin by suppressing multidrug resistance $1 /$ P-glycoprotein (MDR1/P-gp) expression [18]. MiR-19b-3p promotes colon cancer proliferation and oxaliplatinbased chemoresistance by targeting SMAD4 [19]. In the current study, we identified the critical role of miR-744 in the chemoresistance of colorectal cancer and revealed its regulating mechanisms.

MiR-744 has been rarely investigated in CRC, but it has been reported to exert an anti-tumor role in many other cancers. A previous study revealed that the serum miR-744 of gastric cancer patients was significantly higher than the control serum and showed an increasing trend during GC development [10]. For nasopharyngeal carcinoma, miR-744 expression was upregulated compared to normal tissue, and was significantly associated with TNM stage, tumorigenesis and metastasis [20,21]. In pancreatic cancer, expression of miR-744 was markedly upregulated and positively correlated with poor patient survival [9]. All these studies suggest that
miR-744 might promote the development of many cancers. However, the mechanism differs in different cancers. In our work, we found the elevated expression of miR-744 when the HCT116 cells were treated with oxaliplatin, indicating that miR-744 was involved in the chemotherapy response of CRC. Further experiment showed that overexpression of miR-744 conferred HCT116 cells the capacity of antioxaliplatin induced cell death. The chemoresistance role of miR-744 was also investigated in other cancers. In a study of leukemia, miR-744 inhibited the protein level of CCAAT/ enhancer-binding protein delta (CEBPD), which is activated by bortezomib and contributes to bortezomib-induced leukemic cell apoptosis [22]. A high level of plasma miR-744 was found in prostate cancer ( $\mathrm{PCa}$ ) patients who underwent gemcitabine-based chemotherapy. And overexpression of miR-744 in PCa cells induced significant chemoresistance to gemcitabine in vitro [8]. Evidently high expression of miR-744 was found in chemotherapy ineffective group comparing with effective group in breast cancer formalinfixed paraffin-embedded breast cancer tissues [23, 24]. Our work supplied to CRC cell lines to confirm that miR-744 promoted the chemoresistance of CRC cells.

The mechanism of miR-744 mediated chemoresistance was proved to relate to BIN1, which is a well investigated tumor suppressor. CRC cells develop resistance to oxaliplatin through reduced cellular uptake, impaired DNA adduct formation, DNA repair gene alternations and transporter modifications [25-27]. It is reported that inhibition of BIN1 
promoted releasing poly ADP-ribose polymerase 1 (PARP1), which resulted in increased DNA repair activity and allowed gastric cancer cells to acquire cisplatin resistance [28]. Depletion of endogenous BIN1 abolishes cisplatin-induced cell death via activation of c-MYC and PARP1 and sensitize cancer cells to DNA damage [29]. We also found a reduction of BIN1 in oxaliplatin treated cells in the current study, which suggested a similar role of BIN1 in oxaliplatin resistance. The increased miR-744 induced oxaliplatin resistance of CRC cells and downregulated BIN1 in the same time. When we overexpressed BIN1, the miR-744 transfected CRC cells recovered the sensitivity to oxaliplatin. Moreover, miR-744 could directly bind to the 3'-UTR sequences of BIN1 and inhibit the protein level of BIN1, which proved BIN1 to be a direct target of miR-744. Although BIN1 has other functions in the process of many disease, such as membrane trafficking [30], cytoskeleton network [31] and cell cycle regulation[32], its role is also very important in drug resistance of cancer cell.

In conclusion, we have demonstrated that miR-744 expression is upregulated in oxaliplatin treatment. Furthermore, the level of BIN1 is negatively correlated with miR-744 expression and BIN1 is proved to be a direct target of miR-744. Reduction of miR-744 expression may increase chemosensitivity in CRC treatment. Additionally, these findings may be beneficial for predicting drug resistance in patients and designing more effective therapy for colorectal carcinoma patients.

Acknowledgements: The authors wish to thank the First Affiliated Hospital of Xi'an Jiaotong University for providing cell culture support.

\section{References}

[1] SIEGEL RL, MILLER KD, FEDEWA SA, AHNEN DJ, MEESTER RGS et al. Colorectal cancer statistics, 2017. CA Cancer J Clin 2017; 67: 177-193. https://doi.org/10.3322/ caac. 21395

[2] SIEGEL RL, MILLER KD, JEMAL A. Colorectal Cancer Mortality Rates in Adults Aged 20 to 54 Years in the United States, 1970-2014. JAMA 2017; 318: 572-574. https://doi. org/10.1001/jama.2017.7630

[3] MANFREDI S, BOUVIER AM, LEPAGE C, HATEM C, DANCOURT $V$ et al. Incidence and patterns of recurrence after resection for cure of colonic cancer in a well defined population. Br J Surg 2006; 93: 1115-1122. https://doi. org/10.1002/bjs.5349

[4] CALIN GA, CROCE CM. MicroRNA signatures in human cancers. Nat Rev Cancer 2006; 6: 857-866. https://doi. org/10.1038/nrc1997

[5] MICHAEL MZ, SM OC, VAN HOLST PELLEKAAN NG, YOUNG GP, JAMES RJ. Reduced accumulation of specific microRNAs in colorectal neoplasia. Mol Cancer Res 2003; 1 : 882-891.
[6] BRENNECKE J, HIPFNER DR, STARK A, RUSSELL RB, COHEN SM. bantam encodes a developmentally regulated microRNA that controls cell proliferation and regulates the proapoptotic gene hid in Drosophila. Cell 2003; 113: 25-36. https://doi.org/10.1016/s0092-8674(03)00231-9

[7] NURUL-SYAKIMA AM, YOKE-KQUEEN C, SABARIAH AR, SHIRAN MS, SIGNH A et al. Differential microRNA expression and identification of putative miRNA targets and pathways in head and neck cancers. Int J Mol Med 2011; 28: 327-336. https://doi.org/10.3892/ijmm.2011.714

[8] MIYAMAE M, KOMATSU S, ICHIKAWA D, KAWAGUCHI T, HIRAJIMA $S$ et al. Plasma microRNA profiles: identification of miR-744 as a novel diagnostic and prognostic biomarker in pancreatic cancer. Br J Cancer 2015; 113: 14671476. https://doi.org/10.1038/bjc.2015.366

[9] ZHOU W, LI Y, GOU S, XIONG J, WU H et al. MiR-744 increases tumorigenicity of pancreatic cancer by activating Wnt/beta-catenin pathway. Oncotarget 2015; 6: $37557-$ 37569. https://doi.org/10.18632/oncotarget.5317

[10] SONG MY, PAN KF, SU HJ, ZHANG L, MA JL et al. Identification of serum microRNAs as novel non-invasive biomarkers for early detection of gastric cancer. PLoS One 2012; 7: e33608. https://doi.org/10.1371/journal.pone.0033608

[11] SAKAMURO D, ELLIOTT KJ, WECHSLER-REYA R, PRENDEGAST GC. BIN1 is a novel MYC-interacting protein with features of a tumour suppressor. Nat Genet 1996; 14: 69-77. https://doi.org/10.1038/ng0996-69

[12] SAKAMURO D, PRENDEGAST GC. New Myc-interacting proteins: a second Myc network emerges. Oncogene 1999; 18: 2942-2954. https://doi.org/10.1038/sj.onc.1202725

[13] CASSIMERE EK, PYNDIAH S, SAKAMURO D. The cMYC-interacting proapoptotic tumor suppressor BIN1 is a transcriptional target for E2F1 in response to DNA damage. Cell Death Differ 2009; 16: 1641-1653. https://doi. org/10.1038/cdd.2009.98

[14] WANG Y, LIEBERMAN R, PAN J, ZHANG Q, DU M et al. miR-375 induces docetaxel resistance in prostate cancer by targeting SEC23A and YAP1. Mol Cancer 2016; 15: 70. https://doi.org/10.1186/s12943-016-0556-9

[15] VENTURUTTI L, CORDO RUSSO RI, RIVAS MA, MERCOGLIANO MF, IZZO F et al. MiR-16 mediates trastuzum$\mathrm{ab}$ and lapatinib response in ErbB-2-positive breast and gastric cancer via its novel targets CCNJ and FUBP1. Oncogene 2016; 35: 6189-6202. https://doi.org/10.1038/onc.2016.151

[16] LI Y, ZHAO L, LI N, MIAO Y, ZHOU H et al. miR-9 regulates the multidrug resistance of chronic myelogenous leukemia by targeting ABCB1. Oncol Rep 2017; 37: 2193-2200. https://doi.org/10.3892/or.2017.5464

[17] XU K, CHEN G, QIU Y, YUAN Z, LI H et al. miR-503-5p confers drug resistance by targeting PUMA in colorectal carcinoma. Oncotarget 2017; 8: 21719-21732. https://doi. org/10.18632/oncotarget.15559

[18] ZHOU H, LIN C, ZHANG Y, ZHANG X, ZHANG C et al. miR-506 enhances the sensitivity of human colorectal cancer cells to oxaliplatin by suppressing MDR1/P-gp expression. Cell Prolif 2017; 50. https://doi.org/10.1111/cpr.12341 
[19] JIANG T, YE L, HAN Z, LIU Y, YANG Y et al. miR-19b-3p promotes colon cancer proliferation and oxaliplatin-based chemoresistance by targeting SMAD4: validation by bioinformatics and experimental analyses. J Exp Clin Cancer Res 2017; 36: 131. https://doi.org/10.1186/s13046-017-0602-5

[20] FANG Y, ZHU X, WANG J, LI N, LI D et al. MiR-744 functions as a proto-oncogene in nasopharyngeal carcinoma progression and metastasis via transcriptional control of ARHGAP5. Oncotarget 2015; 6: 13164-13175. https://doi. org/10.18632/oncotarget.3754

[21] YU Q, ZHANG F, DU Z, XIANG Y. Up-regulation of serum miR-744 predicts poor prognosis in patients with nasopharyngeal carcinoma. Int J Clin Exp Med 2015; 8: 13296-13302.

[22] CHU YY, KO CY, WANG SM, LIN PI, WANG HY et al. Bortezomib-induced miRNAs direct epigenetic silencing of locus genes and trigger apoptosis in leukemia. Cell Death Dis 2017; 8: e3167. https://doi.org/10.1038/cddis.2017.520

[23] CHEN J, WANG Q, ZHANG W, LI L. [Effect of MTRR gene on apoptosis and autophagy pathways in multiresistant epithelial ovarian cancer]. Zhonghua Fu Chan Ke Za Zhi 2016; 51: 285-292. https://doi.org/10.3760/cma.j.issn.0529567X.2016.04.008

[24] LEIVONEN SK, SAHLBERG KK, MAKELA R, DUE EU, KALLIONIEMI $O$ et al. High-throughput screens identify microRNAs essential for HER2 positive breast cancer cell growth. Mol Oncol 2014; 8: 93-104. https://doi. org/10.1016/j.molonc.2013.10.001

[25] PLASENCIA C, MARTINEZ-BALIBREA E, MARTINEZCARDUS A, QUINN DI, AABD A et al. Expression analysis of genes involved in oxaliplatin response and development of oxaliplatin-resistant HT29 colon cancer cells. Int J Oncol 2006; 29: 225-235. https://doi.org/10.3892/ijo.29.1.225
[26] ARANGO D, WILSON AJ, SHI Q, CORNER GA, ARANES $\mathrm{MJ}$ et al. Molecular mechanisms of action and prediction of response to oxaliplatin in colorectal cancer cells. Br J Cancer 2004; 91: 1931-1946. https://doi.org/10.1038/sj.bjc.6602215

[27] KWEEKEL DM, GELDERBLOM H, GUCHELAAR HJ. Pharmacology of oxaliplatin and the use of pharmacogenomics to individualize therapy. Cancer Treat Rev 2005; 31 : 90-105. https://doi.org/10.1016/j.ctrv.2004.12.006

[28] TANIDA S, MIZOSHITA T, OZEKI K, TSUKAMOTO H, KAMIYA $\mathrm{T}$ et al. Mechanisms of Cisplatin-Induced Apoptosis and of Cisplatin Sensitivity: Potential of BIN1 to Act as a Potent Predictor of Cisplatin Sensitivity in Gastric Cancer Treatment. Int J Surg Oncol 2012; 2012: 862879. https://doi. org/10.1155/2012/862879

[29] PYNDIAH S, TANIDA S, AHMED KM, CASSIMERE EK, CHOE C et al. c-MYC suppresses BIN1 to release poly(ADPribose) polymerase 1: a mechanism by which cancer cells acquire cisplatin resistance. Sci Signal 2011; 4: ra19. https://doi. org/10.1126/scisignal.2001556

[30] MARKS B, MCMAHON HT. Calcium triggers calcineurin-dependent synaptic vesicle recycling in mammalian nerve terminals. Curr Biol 1998; 8: 740-749. https://doi. org/10.1016/s0960-9822(98)70297-0

[31] MEUNIER B, QUARANTA M, DAVIET L, HATZOGLOU A, LEPRINCE C. The membrane-tubulating potential of amphiphysin 2/BIN1 is dependent on the microtubule-binding cytoplasmic linker protein 170 (CLIP-170). Eur J Cell Biol 2009; 88: 91-102. https://doi.org/10.1016/j.ejcb.2008.08.006

[32] WECHSLER-REYA RJ, ELLIOTT KJ, PRENDERGAST GC. A role for the putative tumor suppressor Bin1 in muscle cell differentiation. Mol Cell Biol 1998; 18: 566-575. https://doi. org/10.1128/mcb.18.1.566 